\title{
The mechanism of the deep Bolivia earthquake of June 9, 1994
}

\author{
Masayuki Kikuchi \\ Department of Physics, Yokohama City University, Yokohama, Japan
}

\section{Hiroo Kanamori}

Seismological Laboratory, California Institute of Technology, Pasadena

\begin{abstract}
We analyzed the IRIS broad-band seismograms for the June 9, 1994, Bolivia earthquake, the largest deep-focus earthquake ever recorded. We inverted the record sections with a duration of $330 \mathrm{sec}$ which include $P, p P$ and $s P$ phases, and obtained a dip-slip mechanism: $(\operatorname{dip}(\delta), \operatorname{rake}(\lambda)$, strike $(\phi))=\left(13^{\circ},-4^{\circ}, 1^{\circ}\right)$ or $\left(89^{\circ},-103^{\circ}, 95^{\circ}\right)$, with a scalar moment of $M o=2.9 \times 10^{21}$ $\mathrm{Nm}(M w=8.2)$. This mechanism is similar to that obtained from long-period (175 to $250 \mathrm{sec}$ ) Rayleigh and Love waves: $(\delta, \lambda, \phi)=\left(83^{\circ},-100^{\circ}, 90^{\circ}\right) ; M o=3.0 \times 10^{21}$ $\mathrm{Nm}(M w-8.3)$. The main rupture was preceded by about $10 \mathrm{sec}$ by a cluster of smaller events with a total moment of $1.2 \times 10^{20} \mathrm{Nm}(M w=7.3)$. The main rupture starts at about $20 \mathrm{~km}$ east or northeast of the initial break, and propagates horizontally in the east to northeast direction. The source duration is $40 \mathrm{sec}$, and the rupture dimension is about $40 \mathrm{~km}$. If a fault area of $40 \times 40 \mathrm{~km}^{2}$ is assumed, the stress drop is $110 \mathrm{MPa}$. An inversion of body waves $(P, p P$, and $s P)$ with 6 basis moment tensors yielded a best-fit solution with a $10 \%$ implosive component. This result is of marginal significance and its confirmation would require more detailed information on the structures near the source region, $p P$ bounce points and stations. The observed amplitude of the radial mode, ${ }_{0} S_{0}$, $(1226 \mathrm{sec})$ is consistent with a double couple model with less than $2 \%$ isotropic component.
\end{abstract}

\section{Introduction}

The Bolivia earthquake of June 6, 1994 [O.T.= 00:33:16.1 UT; Location $=\left(13.862^{\circ} \mathrm{S}, 67.540^{\circ} \mathrm{W}\right) ;$ Depth $=637 \mathrm{~km}$ after USGS] is the largest deep-focus earthquake instrumentally recorded. The nature of deep-focus earthquakes, both seismological and physical, has long been debated by many investigators. A comprehensive review on this topic can be found in Frohlich [1989].

It is generally accepted that the radiation pattern of most deep-focus earthquakes can be explained by a double couple source or its combinations without significant isotropic component (i.e. volume change). Honda [1934] demonstrated this (though he did not use the term "double couple") a long time ago; more recently, Stimpson and Pearce [1987] and

Copyright 1994 by the American Geophysical Union.

Paper number 94GL02483

0094-8534/94/94GL-02483\$03.00
Kawakatsu [1991] concluded that the isotropic component is insignificant, less than $10 \%$ of the double couple. This was initially somewhat surprising. Because of the high pressure and temperature in the source region, ordinary brittle failure is not likely to occur and some other mechanisms such as shear instability [Ogawa, 1987] need to be considered. Green and Burnley [1989] and Kirby et al. [1991] proposed transformational faulting as a plausible mechanism of deep-focus earthquakes. In this case a phase transition triggers a rupture, but the main rupture occurs under the ambient shear stress so that no large volume change is expected. Then, considering its geophysical implications [Geller, 1990], it would be important to determine how small the isotropic component is.

The rupture geometry of deep-focus earthquakes is generally interpreted in terms of the stress in the subducting slab [Isacks' and Molnar, 1971]. Fukao [1973] argued, on the basis of energetics, that the rupture is more likely to occur on the more steeply dipping plane of the two nodal planes. The choice of fault plane is not explicit in Kirby et al.'s [1991] model, but the geometry of the fault plane would provide a useful constraint on the physical mechanism of deep-focus earthquakes.

The stress drop of deep-focus earthquakes is generally considered to be larger than that of shallow earthquakes [e.g. Mikumo, 1971; Fukao and Kikuchi, 1987], but the stress drop determination is usually subject to large uncertainties and large variations have been reported.

The very large size of the Bolivia earthquake and the availability of many high-quality broadband seismograms provide a good opportunity to investigate these features in some detail. Here we present results on the geometry of the rupture plane, stress drop and isotropic component of the June 9, 1994, Bolivia earthquake obtained from the global IRIS broadband data.

\section{Point source inversion}

Figure 1a shows the IRIS broadband seismograph stations that recorded the Bolivia earthquake at the epicentral distances between $30^{\circ}$ and $100^{\circ}$. Figure $1 \mathrm{~b}$ shows an example of the $P$ wave displacement record. For deep-focus earthquakes, the displacement record of direct $P$ wave is a good approximation of the source time function [Kikuchi and Ishida, 1993]. Figure 1b shows a cluster of three distinct subevents during the first 10 sec. Figure ic shows the vertical component of $P$-wave displacement records obtained from the stations shown in Figure 1a. The records are displayed so that the beginnings of the small initial event are lined up at time 0 . Then the onsets of the main rupture at 10 to $13 \mathrm{sec}$ reveal a systematic azimuthal variation, indicating an eastward offset of the initiation point of 


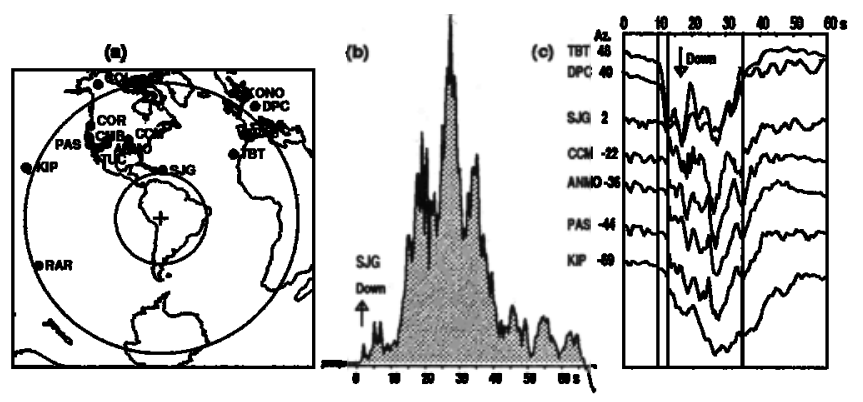

Figure 1. (a) IRIS broadband seismograph stations. Small and large circles indicate the epicentral distances of $30^{\circ}$ and $90^{\circ}$ respectively. (b) $P$-wave displacement at SJG. (c) Azimuthal dependence of $P$-wave displacement.

the main rupture by about $15 \mathrm{~km}$ relative to the initial break.

The main part of the time function indicates a complex multiple shock sequence with several subevent clusters. First we determined the overall mechanism using the inversion method of Kikuchi and Kanamori [1991]. We windowed record sections with a duration of $330 \mathrm{sec}$ and a sampling time of $1 \mathrm{sec}$ from the vertical component of displacement records at 14 stations. These records include $P, p P$ and $s P$ phases. We inverted the data for a single point source which is constrained to be a double couple. The standard Jeffreys-Bullen model was then assumed for the earth's structure. Using a trapezoidal source time function with a duration of $32 \mathrm{sec}$, we obtained a dip-slip mechanism with a nearly horizontal fault plane: (dip( $\delta$ ), rake $(\lambda)$, strike $(\phi))=\left(13^{\circ},-4^{\circ}, 1^{\circ}\right)$ or a nearly vertical fault plane: $(\delta, \lambda, \phi)=\left(89^{\circ},-103^{\circ}, 95^{\circ}\right)$, with a scalar moment of $2.2 \times 10^{21} \mathrm{Nm}$. This mechanism is similar to that we obtained from long-period (175 to $250 \mathrm{sec}$ ) Rayleigh and Love waves $(\delta, \lambda, \phi)=\left(83^{\circ},-100^{\circ}, 90^{\circ}\right), M o=3.0 \times 10^{21} \mathrm{Nm}(M w=8.3)$ and the routine CMT solutions obtained by Harvard, Caltech and Earthquake Research Institute of the University of Tokyo.

\section{Initial rupture}

Figures $1 b$ and $1 c$ indicate an initial stage of rupture propagation preceding the main rupture by about $10 \mathrm{sec}$. At least three subevents can be identified. The polarity of $P$ waves indicates that the mechanism of these subevents is similar to that of the main rupture, but a closer inspection of the record at RAR suggests a slightly different mechanism for the third subevent.

We inverted the initial $10 \mathrm{sec}$ of the $P$ waves into the source time function. The total moment is $1.2 \times 10^{20} \mathrm{Nm}(M w=7.3)$, about $5 \%$ of the main rupture, but still significant. The source parameters are given in Table 1, where the subevents are denoted by $I-1,2,3$.

\section{Fault plane and rupture extent}

It is not always easy to distinguish the fault plane from the auxiliary plane, especially for deep-focus earthquakes for which neither field evidence nor geodetic data are available. To fully understand the physical mechanism of trigger and rupture of deep-focus earthquakes it is important to determine the geometry of the fault plane. To this end, we performed the following tests. First we examined the vertical extent of rupture using waveform correlation. Let $x_{j}(t)$ denote the observed waveform at the $j$-th station, and $w_{j}(t, x)$ denote the synthetic
Table 1. Source parameters

\begin{tabular}{lcccc}
\hline$\#$ & time(s) & distance(km) & azimuth( $\left(^{\circ}\right)$ & $M_{0}(\mathrm{Nm})$ \\
\hline I 1 & $0-2$ & 0 & - & $0.23 \times 10^{20}$ \\
I 2 & $3-7$ & 0 & - & 0.46 \\
I 3 & $6-10$ & 0 & - & 0.52 \\
& & sub-total & & $1.15 \times 10^{20}$ \\
M1 & $11-19$ & 20 & 70 & $4.92 \times 10^{20}$ \\
M2 & $17-29$ & 30 & 60 & 10.76 \\
M3 & $24-34$ & 30 & 45 & 6.53 \\
M4 & $31-43$ & 40 & 60 & 6.97 \\
& & sub-total & & $29.23 \times 10^{20}$ \\
\hline
\end{tabular}

$(\delta, \lambda, \phi)=(13,-4,1)$ except for $13,(23,-59,309)$ for 13

wavelet generated by a double couple at $x$ and observed at the $j$-th station, then the correlation function $\Psi(\tau, x)$ is defined by:

$$
\Psi(\tau, \mathrm{x})=\sum_{j} \int x_{j}(t) w_{j}(t-\tau, \mathrm{x}) d t
$$

For each $\mathbf{x}$, we varied $\tau$ to obtain the maximum correlation $\Psi_{\max }(\mathrm{x})$. Here we used a narrow (10sec) source time function for a dip-slip mechanism obtained above, set up a grid network on the vertical nodal plane, moved a point source over the entire grid points, computed a correlation coefficient at each grid point, and contoured the result in the form of a correlation map as shown in Figure 2a. If the real subevents are distributed on the vertical nodal plane, correlation will be good or poor depending on whether or not the model subevent is at the same depth as the real subevent. This would result in a smeared pattern on the correlation map. In contrast, if the real subevents are distributed on the horizontal plane, the correlation will be good only for a very narrow depth range. As Figure 2a shows, the actual correlation map has a sharp peak at a depth of $650 \pm 10 \mathrm{~km}$, strongly suggesting that the rupture plane is horizontal. The absolute depth depends on the earth's structure used in inversion, but the range does not depend on the model.

In the second test, we inverted the data with multiple point sources constrained to be either on the horizontal or vertical plane. The inversion for the horizontal distribution yielded a better fit to the data than that for the vertical plane. Also, when the point sources are constrained to be on the vertical plane, the inversion yielded a distribution of point sources along a nearly horizontal line on the vertical plane as shown in Figure $2 b$. This suggests that rupture occurred on the horizontal plane.

Once the fault plane is identified, we can achieve a better resolution of the source location using a higher sampling rate (2 $\mathrm{Hz}$ ) and a shorter time-window that includes only $P$ phase. We inverted the observed $P$ waves during the first $90 \mathrm{sec}$ using point sources with the mechanism fixed at $(\delta, \lambda, \phi)=\left(13^{\circ},-4^{\circ}\right.$, $1^{\circ}$ ) distributed on the shallow-dip fault plane. The result is
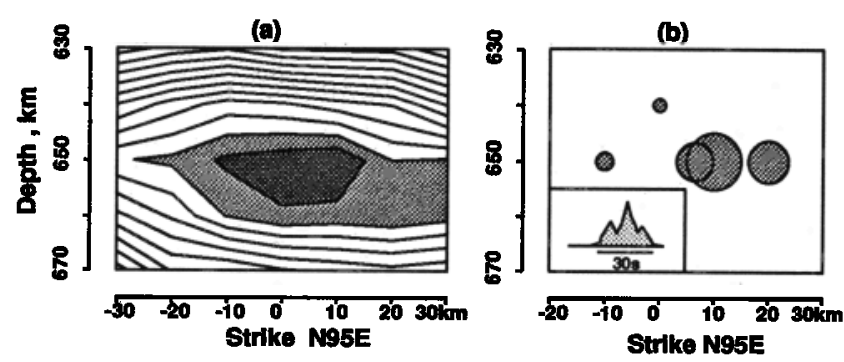

Figure 2. (a) Correlation function $\Psi \max (x)$ on the vertical $P$-wave nodal plane. (b) Subevents constrained on the vertical plane. The inset shows the source time function. 
shown in Figure 3. The total seismic moment is $M o=2.9 \times 10^{21}$ $\mathrm{Nm}(M w=8.2)$, and the source duration is about $40 \mathrm{sec}$.

The main rupture starts at about $20 \mathrm{~km}$ east or northeast of the initial break, and propagates in the northeast direction over a fan-shaped area. However, the resolution of location in the NS direction is poor because of the lack of stations in the south, so that the possibility of a ribbon-shaped source striking in the east to northeast direction with very limitted (less than $10 \mathrm{~km}$ ) horizontal and vertical extents is not ruled out. The details of the source parameters are given in Table 1 , where subevents are denoted by $M-1,2,3,4$.

The rupture area is small for an earthquake with $M w=8.2$. This results in a very large average stress drop. The fault area inferred from Figure 3 is about $S=40 \times 40 \mathrm{~km}^{2}$ at most, and the stress drop averaged over this area is $\Delta \sigma=2.4 \times M o S^{-1.5}=$ $110 \mathrm{MPa}$. It is also remarkable that the apparent rupture velocity is very slow, about $1 \mathrm{~km} / \mathrm{s}$. This slow rupture seems to be consistent with Green and Burnley's [1989] prediction.

\section{Isotropic component}

We attempted a moment-tensor inversion with 6 basis moment tensors defined by Kikuchi and Kanamori [1992] using the data set with a $330 \mathrm{sec}$ duration. The best-fit moment tensor thus derived has an isotropic component with $I=$ $-(2.2 \pm 0.3) \times 10^{20} \mathrm{Nm}$, where the negative sign means "implosion". The deviatoric component is given by a double couple with the mechanism: $(\delta, \lambda, \phi)=\left(8^{\circ},-12^{\circ}, 351^{\circ}\right)$, or $\left(88^{\circ},-98^{\circ}\right.$, $93^{\circ}$ ) and $M o=2.2 \times 10^{21} \mathrm{Nm}$. The mechanism diagram and the trapezoidal source time function with a duration of $32 \mathrm{sec}$ are shown in Figures $4 a$ and $b$. The scalar moment of the isotropic component amounts to nearly $10 \%$ of that of the double couple.

The main evidence for the isotropic component came from the ratio of $p P$ to $P$ phases. A double couple source yields $P$ and $p P$ phases either in the upward (e.g. RAR) or in downward polarity (e.g. PAS) depending on the position of the stations on the focal sphere. In contrast, the polarity of $P$ and $p P$ for an isotopic source does not depend on the position of stations. Thus the variation of $p P / P$ ratio at different stations carries the information on the ratio of double couple to isotropic component. The absence of $s P$ phase from an isotropic source also contributes to distinguishing a double couple from an isotropic
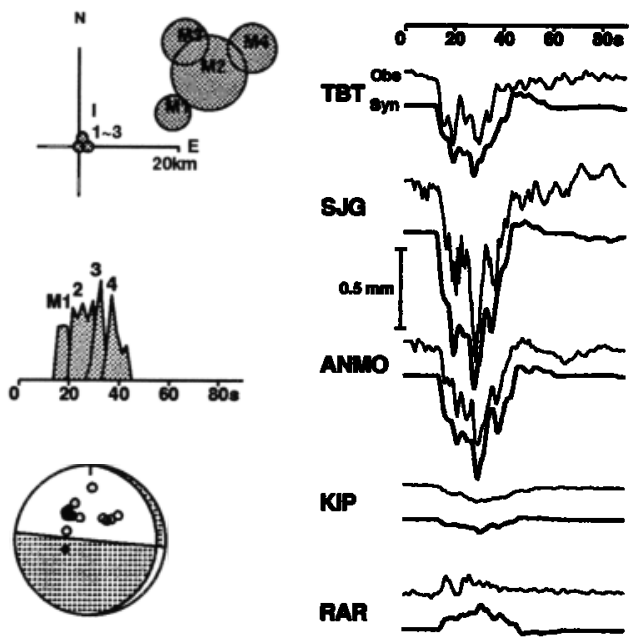

Figure 3. The main rupture process. Left top: spatial distribution of subevents on the shallow-dip plane; middle: moment-rate function; bottom: fault mechanism; Right: waveform comparison.

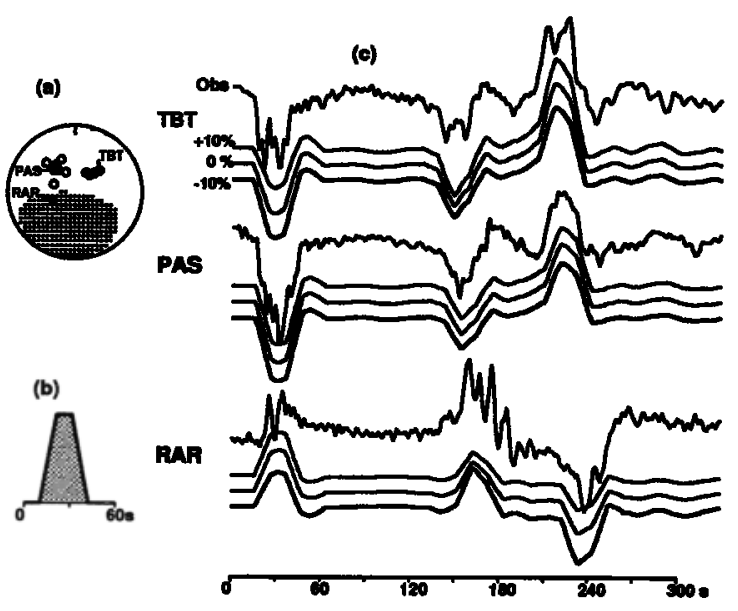

Figure 4. (a) Mechanism diagram for double couple (DC) $+10 \%$ implosive source. (b) Source time function. (c)Waveform comparison in three cases: explosion (denoted by $+10 \%$ ), pure DC (0\%) and implosion $(-10 \%)$.

source. Figure $4 c$ shows the comparison of the observed and synthetic waveforms for sources with $+10 \%$ (explosion), $0 \%$ (pure double couple) and $-10 \%$ (implosion) isotropic component. The normalized variances for these three cases are $.320, .311$ and .306 , respectively. Thus the effect of the isotropic source on the variance reduction is not large, but it is still visible in Figure $4 c$. This result may depend on the structure of the $p P$ bounce point. Our test using an oceanic crust and continental crust for the bounce point shows that the effect of the crustal structure on $p P / P$ ratio is not large enough to change the above conclusion. There is also some trade-off between the mechanism and the amount of isotropic component. Since very few stations are available in the southern azimuth, this trade-off is difficult to examine further. Considering these and all the inevitable assumptions made for modelling, the implosive component obtained above is considered of marginal significance.

We made another test of the isotropic component using the radial mode $S_{0}$ with the period of $1226 \mathrm{sec}$. This test is especially useful for the Bolivian earthquake because its mechanism suggests very little excitation of the ${ }_{0} S_{0}$ mode. A vertical (or horizontal) fault, i.e. $\delta=90^{\circ}$, does not excite $0 S_{0}$ mode because of its particular geometry. In contrast, an isotropic source excites $S_{0}$ mode very efficiently. Thus the amplitude of ${ }_{0} S_{0}$ mode is a good measure of the isotropic component. Figure 5 shows the spectral peak of ${ }_{0} S_{0}$ mode observed at Pasadena for a time window of 0 to 4.63 days after the earthquake. Also shown is the spectrum obtained from a time-domain stack of 13 IRIS stations, which agrees with that from Pasadena. This good agreement suggests that the spectral amplitude is accurately determined. Figure 5 compares these observations with the spectra computed for the HRV CMT solution: $(\delta, \lambda, \phi)=$ $\left(82^{\circ},-93^{\circ}, 90^{\circ}\right)$, and $M o=3 \times 10^{21} \mathrm{Nm}$, and for an isotropic source with a dipole moment of $3 \times 10^{20} \mathrm{Nm}$. The synthetic spectra were computed using the PREM velocity and $Q$ models.

Figure 5 suggests that the double couple mechanism could explain the observed amplitude of ${ }_{0} S_{0}$, and no isotropic component is necessary. However, some trade-off exists between the amount of isotropic component and the mechanism. The amplitude of the ${ }_{0} S_{0}$ mode excited by a nearly vertical fault depends primarily on $\delta$. A change in rake of $\pm 5^{\circ}$ from $-93^{\circ}$ results in only $0.4 \%$ change in the amplitude. In contrast, if $\delta$ is $86^{\circ}$, the amplitude will be halved; if $\delta$ is reduced to $73^{\circ}$, the 


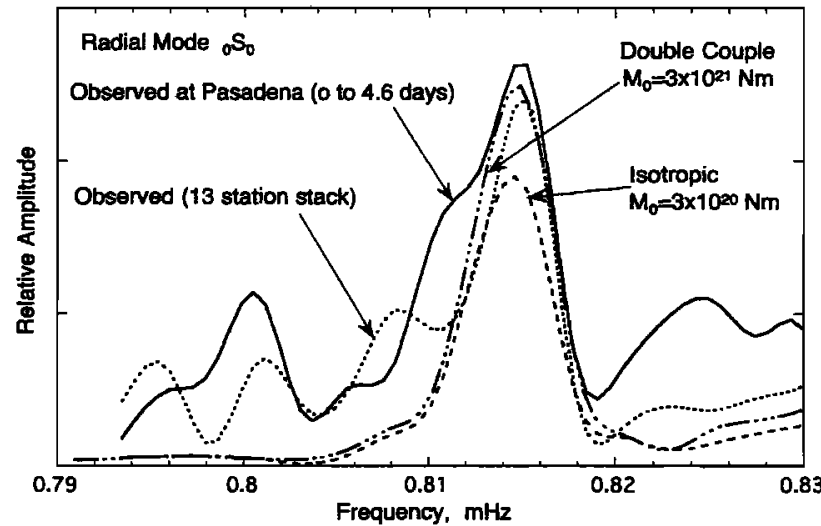

Figure 5. Spectral peaks of ${ }_{0} S_{0}$ mode obtained from PAS seismogram and a 13 station stack. Theoretical spectra for a double couple and isotropic source are compared.

amplitude will double. Considering the phase (a normal fault and an explosive source excite $S_{0}$ mode in phase), this means that if $\delta=86^{\circ}$, a $7 \%$ explosive source is required, and if $\delta=73^{\circ}$, a $12 \%$ implosive source will be required to explain the observed amplitude of the $S_{0}$ mode. However, the dip angle determined from long-period waves is within a very narrow range, 81 to $83^{\circ}$, so that the allowable amount of isotropic component at long period is probably $\pm 2 \%$, even if a $10 \%$ uncertainty is attached to $M_{0}$.

We conclude from the analysis of both body waves and $0 S_{0}$ mode that the isotropic component of the Bolivia earthquake, if it exists, is no more than $10 \%$ of the double couple component, which agrees with the conclusion on deep-focus earthquakes in general. There is some evidence from the $p P / P$ ratios that the source involved a $10 \%$ implosive component at least for the first $50 \mathrm{sec}$. Confirmation of this may require more detailed information on the structures near the source region, $p P$ bounce points and stations.

\section{Discussion}

The rupture geometry of the Bolivia earthquake is very different from those determined for other large deep-focus earthquakes. Fukao and Kikuchi [1987] analyzed large deepfocus earthquakes including the Nov. 9, 1963, western Brazil, the Aug.15, 1963, Peru-Bolivia and the July 31, 1970, Colombia earthquakes using the WWSSN records and found that the fault planes of most dip-slip earthquakes are steeper than the auxiliary planes. In contrast, the fault plane of the Bolivia earthquake is almost horizontal. Also the horizontal projections of both P- and T-axes of the Bolivia earthquake are parallel to the regional trend of the subduction zone. For most deep-focus earthquakes, they are perpendicular to it. This difference could be due to the geometry of the subducting slab in this region. Unfortunately, the slab geometry in this region is uncertain because of the absence of intermediate-depth activity, and a tomographic imaging would be necessary to determine it.

If the implosive component determined from body waves is real, it may represent volume reduction due to phase transition which triggered rupture in a manner described by Kirby et al. [1991]. Then the question is why the implosive component vanished at long period. One possibility is that there was volume increase during faulting. Under the very high stress, fault motion is likely to cause melting which may result in volume increase cancelling the volume reduction due to phase transition. This interpretation is somewhat speculative and must await further corroborative evidence

Acknowledgements. We benefitted from discussions with Don Anderson, Tom Heaton, and Shingo Watada. This research was partially supported by the Grant-In-Aid for Scientific Research No.04452067 from the Ministry of Education, Japan, and by the National Science Foundation Grant EAR-9303804, USA. Contribution No. 5430, Division of Geological and Planetary Sciences, California Institute of Technology, Pasadena, California 91125.

\section{References}

Frohlich, C., The nature of deep-focus earthquakes, Ann. Rev. Earth Planet. Sci., 17, 227-254, 1989.

Fukao, Y., Seismological evidence for selectivity in slip planes under down-dip extension or compression, Geofisica International, 13, 223-242, 1973.

Fukao, Y., and M. Kikuchi, Source retrieval for Mantle earthquakes by iterative deconvolution of long-period P-waves, Tectonophysics, 144, 249-269, 1987.

Geller, R.J., Metastable phases confirmed, Nature , 347, 620-621, 1990.

Green, H.W., and P.C. Bumley, A new self-organizing mechanism for deep-focus earthquakes, Nature, 341, 733-737,1989.

Honda, $H$., On the amplitudes of the $P$ and $S$ waves of deep earthquakes, Geophys. Mag. , 8, 153-164, 1934.

Isacks, B., and Molnar, P., Distribution of stresses in the descending lithosphere from a global survey of focal-mechanism solutions of mantle earthquakes, Rev. Geophys. Space Phys., 9, 103-174, 1971.

Kawakatsu, H., Insignificant isotropic component in the moment tensor of deep earthquakes, Nature, 351, 50-53, 1991.

Kikuchi, M., and Ishida, M., Souce retrieval for deep local earthquakes with broadband records, Bull. Seism. Soc. Am., 83, 1855$1870,1993$.

Kikuchi, M., and Kanamori, H., Inversion of complex body wavesIII, Bull. Seism. Soc. Am. 81, 2335-2350, 1991.

Kirby, S.H., W.B. Durham, and L.A. Stern, Mantle phase changes and deep-earthquake faulting in subducting lithosphere, Science, 252, 216-225, 1991.

Mikumo, T., Source process of deep and intermediate earthquakes as inferred from long-period $P$ and $S$ waveforms, 2. Deep-focus and intermediate-depth earthquakes around Japan, J. Phys. Earth, 19. 303-320, 1971.

Ogawa, M., Shear instability in a visco-elastic material as the cause of deep focus earthquakes, J. Geophys. Res., 92, 13801-13810, 1987.

Stimpson, I.G., and Pearce, R.G., Moment tensors and source processes of three deep Sea of Okhotsk earthquakes, Phys. Earth Planet. Inter. , 47, 107-124, 1987.

M. Kikuchi, Dept. Physics, Yokohama City University, Yokohama 236, Japan. (e-mail: kikuchi@yokohama-cu.ac.jp)

H. Kanamori, Seismological Laboratory, California Institute of Technology, Pasadena, CA 91125. (e-mail: hiroo@seismo.gps.caltech.edu)

(Received July 29, 1994; revised August 30, 1994; accepted September 14, 1994.) 